\title{
Stress Energy Quantum Tensor : Linear Approximation of the Einstein's Equations and Equivalence with the Klein-Gordon's equation
}

\author{
Roman Baudrimont \\ Entro- $\pi$ Fluid Group \\ roman.baudrimont@entropyfluid.yo.fr I RomanBaudrimont.cd@gmail.com
}

\begin{abstract}
Our goal in this paper is to study the relationship between the linear approximation of Einstein's equations to the Klein-Gordon's equation. The part one presents what is the Klein-Gordon's equation and the integration of the theory of quantum information in it. The Part two deals with the stress energy quantum tensor, wherein the detail I linearized gravity of Einstein equation, and wherein I develop the stress energy quantum tensor quantum from the equivalence the linearized gravity of Einstein's equation and the Schrödinger equation relativistic described by Klein-Gordon's equation.
\end{abstract}

Keysword: Stress Energy Tensor, Klein-Gordon's Equation, Quantum Mechanics, Quantum Field Theory

\section{Part 1 - Klein-Gordon's equation.}

Part A - Introduction to the Klein-Gordon's equation.

The Klein-Gordon's equation ${ }^{[1]}$ is a relativistic version of the Schrödinger equation describing massive particles of zero spin and, in our case, no electric charge. So I'm here to make a point of this equation.

First, write the Schrödinger equation:

$$
i \hbar \frac{\partial}{\partial t} \psi(\vec{r}, t)=\widehat{H} \psi(\vec{r}, t)
$$

With $\widehat{H}$ the Hamiltonian operator:

$$
\widehat{H}=-\frac{h^{2}}{2 m} \Delta+V(\vec{r}, t)
$$

It is noted that the Hamiltonian (2) gives the total energy of the system with the reduced Planck constant $h$ corresponding to the pulse, $-h^{2} \Delta /(2 m)$ corresponds to the kinetic energy, $V(\vec{r}, t)$ potential energy of the system. The delta $(\Delta)$ is a Laplacian.

It will from the invariant relativistic giving the energy of a single particle. In special relativity, the equation is as follows:

$$
E^{2}=p^{2} c^{2}+m^{2} c^{4}=\vec{p} \cdot \vec{p}+m^{2} c^{4}
$$

We now from the correspondence principle (where the quantum behavior of a system can be reduced to behavior of classical physics). We then have two principles of correspondence:

$$
\begin{gathered}
E=i \hbar \frac{\partial}{\partial t} \\
E^{2}=p^{2} c^{2}+m^{2} c^{4}=\vec{p} \cdot \vec{p}+m^{2} c^{4}
\end{gathered}
$$


With $\vec{\nabla}$ the operator nabla.

We then obtain the following equation:

$$
\begin{gathered}
\vec{p}=i \hbar \vec{\nabla} \\
-h^{2} \frac{\partial^{2}}{\partial t^{2}} \psi(\vec{r}, t)=-h^{2} c^{2} \nabla^{2} \psi(\vec{r}, t)+m^{2} c^{4} \psi(\vec{r}, t)
\end{gathered}
$$

That can be written in the following form:

$$
\Delta \psi(\vec{r}, t)-\frac{1}{c^{2}} \frac{\partial^{2}}{\partial t^{2}} \psi(\vec{r}, t)=\frac{m^{2} c^{2}}{h^{2}} \psi(\vec{r}, t)
$$

With $\Delta=\nabla^{2}$. Finally, we have the following equations :

$$
\left(\Delta-\frac{1}{c^{2}} \frac{\partial^{2}}{\partial t^{2}}\right) \psi(\vec{r}, t)=\frac{m^{2} c^{2}}{h^{2}} \psi(\vec{r}, t)
$$

Here the Klein Gordon equation. However, the latter problem when we want to calculate the probability density. When calculating the probability density, one is faced with a major problem. Indeed, we have:

$$
\rho=\frac{i \hbar}{2 m c^{2}}\left(\psi^{*} \frac{\partial \psi}{\partial t}-\psi \frac{\partial \psi^{*}}{\partial t}\right)
$$

More synthetically, if calculation starting from a single wave function, described as follows, with $A$ is the amplitude of the wave, and $\vec{p}$ the pulse vector ${ }^{[2]}$ :

$$
\psi(\vec{r}, t)=A e^{i(E t-\vec{p} \cdot \vec{r})}
$$

And we calculate the following probability density:

$$
\rho=i\left(\psi^{*} \frac{\partial \psi}{\partial t}-\psi \frac{\partial \psi^{*}}{\partial t}\right)
$$

We get this:

$$
\rho=2 E A^{2}
$$

The expression of the probability density then depends entirely on the energy $E$. Since energy can be negative, this suggests that the probability density can be as negative. An equation that described must then be used as the Schrödinger equation, an equation of first order in the derivatives of the time for which the probability density is positive definite, thus respecting the principles of relativity and quantum mechanics, Dirac equation.

However, quantum physics said probability computation can be done by simply calculating the norm in the square wave function. In this case, the problem regarding the possible negativity of the probability density is set. Now we will see that it is possible to integrate quantum information to the Klein-Gordon's equation. 
Part B - Klein-Gordon's equation and quantum information.

Before calculating theory of quantum information, we will start on the equation of De Broglie wave, in which the wave propagates along the axis of $z^{[3]}$.

$$
\psi(\vec{r}, t)=\psi_{0} e^{i(\omega t-k z)}
$$

One can then define the electric field given by the following equation (14) ${ }^{[3]}$ :

$$
\bar{E}(\vec{r}, t)=\bar{E}_{0} e^{i(\omega t-k z)}
$$

The vector $\bar{E}_{0}$ is given by the following equation:

$$
\bar{E}_{0}=E_{0} \hat{e}_{x}+E_{0} \hat{e}_{y}
$$

It is noted that $\bar{E}_{0}$ is a complex number which defines the polarization of the wave.

As part of the theory of quantum information, we can use the above formula for defining the polarization of the wave to change the Klein-Gordon's equation and thus adapt to quantum information. One example would be to study the position / motion of a particle by defining a probability amplitude of the different possible polarizations of the particle. Thus, if we write ${ }^{[4]}$ :

$$
|\psi\rangle=\alpha|a\rangle+\beta|b\rangle
$$

We could generalize as a complex function, for which the probability amplitude given by the complex $\alpha$ and $\beta$ depends on the vecto $\vec{r}$ and $t$, like so:

$$
|\psi(\vec{r}, t)\rangle=\alpha(\vec{r}, t)|a\rangle+\beta(\vec{r}, t)|b\rangle
$$

What we have above is a complex probabilistic function vector value whose complex probabilistic $\alpha(\vec{r}, t)$ and $\beta(\vec{r}, t)$ functions. We have with the Klein-Gordon's equation :

$$
\left(\Delta-\frac{1}{c^{2}} \frac{\partial^{2}}{\partial t^{2}}\right)|\psi(\vec{r}, t)\rangle=\frac{m^{2} c^{2}}{h^{2}}[\alpha(\vec{r}, t)|a\rangle+\beta(\vec{r}, t)|b\rangle]
$$

This allows us to obtain a relativistic equation the Schrödinger wave function incorporating quantum information.

Moreover, the probability will always be positive. Indeed, the calculation of the probability of a state being performed by the standard square complex probabilistic vector valued functions, the latter will always be positive, we can write as follows:

$$
\begin{aligned}
& 0<|\alpha(\vec{r}, t)|^{2}<1 \\
& 0<|\beta(\vec{r}, t)|^{2}<1
\end{aligned}
$$

So that the following property is verified:

$$
\||\psi(\vec{r}, t)\rangle \|^{2}=|\alpha(\vec{r}, t)|^{2}+|\beta(\vec{r}, t)|^{2}=1
$$

It is important to note that the following function is used to define the movement of particles without spin. However, the formulation that we have done previously to associate the movement of particles in quantum information. 


\section{Part 2 - Stress energy tensor.}

\section{Part A - Linear approximation of stress energy tensor.}

A first formulation of Einstein's equations is as follows ${ }^{[5]}$ :

$$
R^{i j}=\frac{16 \pi G}{c^{4}}\left[\Gamma^{i j}-\frac{1}{2} g^{i j} \Gamma\right]
$$

With $R^{i j}$ the Ricci tensor and $\Gamma^{i j}$ stress energy tensor.

The metric tensor through a gravity field is written by the following equations ${ }^{[4]}$ :

$$
\begin{aligned}
& g_{i j}=\eta_{i j}+h_{i j} \\
& g^{i j}=\eta^{i j}-h^{i j}
\end{aligned}
$$

When $\left|h_{i j}\right| \ll 1$, one can obtain a linear approximation of Einstein's equations given by the following formula ${ }^{[5]}$ :

$$
\left(\Delta-\frac{1}{c^{2}} \frac{\partial^{2}}{\partial t^{2}}\right) h^{i j}=\frac{16 \pi G}{c^{4}}\left[\Gamma^{i j}-\frac{1}{2} \eta^{i j} \Gamma\right]
$$

From wich we obtain the values of the metric tensors :

$$
\begin{aligned}
& g_{i j}=\eta_{i j}+\frac{2 G M}{c^{2} r} \operatorname{diag}(1,1,1,1) \\
& g^{i j}=\eta^{i j}-\frac{2 G M}{c^{2} r} \operatorname{diag}(1,1,1,1)
\end{aligned}
$$

then we can get $h=\eta^{i j} h_{i j}$, considering that the following equation ${ }^{[6]}$ :

$$
\left(\Delta-\frac{1}{c^{2}} \frac{\partial^{2}}{\partial t^{2}}\right) h(\vec{r}, t)=-\frac{8 \pi G}{c^{4}} \Gamma(\vec{r}, t)
$$

Small note: The signed negative now in the right side of the equation does not mean that there is a negative energy. In fact, the tensor represents the attraction while the dot represents repulsion. It is just a distinction between the tensor and the scalar form of equation.

To recall, the equation of the Newtonian gravitation writing is as follows:

$$
\vec{F}=-G \frac{m M}{r^{2}} \vec{u}
$$

However, the stress energy tensor in scalar form isn't a vector, but a scalar ! So we will remove it and we will consider the scalar rating and tensor notation represents the same concept, an attraction. We will rewrite the equation of the stress energy tensor in its linear formalism as follows :

$$
\left(\Delta-\frac{1}{c^{2}} \frac{\partial^{2}}{\partial t^{2}}\right) h(\vec{r}, t)=\frac{8 \pi G}{c^{4}} \Gamma(\vec{r}, t)
$$

We will keep this form for the following sections we will deal with. 
Part B - Stress energy quantum tensor from the Klein-Gordon's equation.

Part B.1 - Equivalence between the small deviation of the Minkowski metric and the quantum wave function.

From the above equations written, we can define an equivalence between the curvature "scalar" granted by the Laplacian of $h(r, t)(31)$ and also the relativistic version of the Schrödinger equation developed by Klein Gordon (9).

$$
\left(\Delta-\frac{1}{c^{2}} \frac{\partial^{2}}{\partial t^{2}}\right) h(\vec{r}, t)=\left(\Delta-\frac{1}{c^{2}} \frac{\partial^{2}}{\partial t^{2}}\right) \psi(\vec{r}, t)
$$

Or, for simplicity, we get:

$$
h(\vec{r}, t)=\psi(\vec{r}, t)
$$

For this equality may exist, one must consider that the linearized gravity of Einstein's equations given by the disturbance $h(\vec{r}, t)$ (which $h^{i j}$ is the low deflection of $\eta_{i j}$ ) is equivalent to the complex function $\psi(\vec{r}, t)$ defining the quantum state of a system with an amplitude probability.

An effective way to establish this equivalence principle can be explained by the so-called small extent. It is a technique used to measure the average value of an observable (or in other words, a physical quantity such as the position or energy) of a quantum system in not disrupting thereof negligibly. Thus, one would find the equivalence between a small perturbation (linearized gravity) of the Einstein equation and the small perturbations occurring during measurement of a quantum system.

It is also noteworthy that the wave function is used to describe the motion of a wave, in the same manner as Newtonian approximation of Einstein equation to calculate the movement of the gravitational waves. Consider his gravitational waves as disturbances of space-time, the latter being generally flat, except that considers its perturbations as low. This means that the movement of gravitational and comparable to that of a particle comprising as a wave relativistic.

Part B.2 - Stress energy quantum tensor.

From the equivalence defined above, we can define the following equation:

$$
\frac{8 \pi G}{c^{4}} \Gamma(\vec{r}, t)=\frac{m^{2} c^{2}}{h^{2}} \psi(\vec{r}, t)
$$

By isolating the pressure, we get:

$$
\Gamma(\vec{r}, t)=\frac{m^{2} c^{6}}{8 \pi G h^{2}} \psi(\vec{r}, t)
$$

We notice when we find the constant $K$ of Einstein's equation $G_{i j}=K \cdot T_{i j}$. We can then write the following formulation:

$$
\Gamma(\vec{r}, t)=K^{-1} \frac{c^{2} m^{2}}{h^{2}} \psi(\vec{r}, t)
$$


If we calculated and are measuring the wave function, we would find that this pressure is not local, with greater or lesser presence probabilities according to the surface area we choose.

However, I want to clarify one important thing: This equation may not be valid to describe a single particle. However, quantum field theory, it can be used to describe a set of zero spin particle.

Suppose we calculate the pressure $\Gamma(\vec{r}, t)$ of an electron. Considering that :

$$
\begin{gathered}
h \approx 1,054571 \times 10^{-34} \mathrm{~J} . \mathrm{s} \\
c \approx 299792458 \mathrm{~m} \cdot \mathrm{s}^{-1} \\
K^{-1}=\frac{c^{4}}{8 \pi G} \approx 4,815612 \times 10^{42} \mathrm{~N} \\
m_{\text {électron }} \approx 9,109382 \times 10^{-31} \mathrm{~kg}
\end{gathered}
$$

We have :

$$
\Gamma_{\text {électron }}(\vec{r}, t) \approx 3,229379 \times 10^{67} \psi(\vec{r}, t)
$$

This means that the pressure of an electron (without calculating the wave function) is about $10^{67}$ !

\section{Part 3 - Conclusion.}

In Part 1, we saw that the Klein-Gordon's equation is a relativistic version of the Schrödinger equation for particles without spin. This equation, second order, some problems, including one very important: the probability density can be negative.

We found a simple solution was to consider the psi function as a complex probabilistic function vector value, which we had reformed under the equations of the theory of quantum information. Thus, by calculating the probability, we are forced to standardize and squaring the probability amplitude, thus solving the problem of possible negativity of probability density.

In section 2, we saw the formulation of the linear approximation (or linearized gravity) of the Einstein equation, wherein the equation is closer to Newtonian dynamics.

We then found a match between the wave function of quantum physics, and low deflection previously defined by calculating the linear approximation of Einstein equation. This equivalence suggests that, in some cases, the description of a gravitational wave reduced to a flat space-time (or in which the curvature of the space-time is not important) is equivalent to the movement of a particle behave like a relativistic wave.

From this equivalence, we described an important equation: The stress energy tensor quantum. We discover that it is not local because it depends on the wave function. The tensor becomes nonlocal.

When calculating the pressure for a particle (an electron), we note that it is very high, on the order of ten to the 67 . Is that a mathematical error? Or not we know enough about the subatomic world?

To these questions I try to answer by finishing the article, when I have calculated from the new physical equivalence entropy. I then try to find the formula for Erik Verlinde linking entropy Newtonian gravity. 


\section{Part 4 - References.}

[1] J.F. Fortin, P.O. Genest, J.F. Laprise, S. Marchand, V. Bérubé et S. Bégin (2003), Les outils de base de la physique des particules, Luc Marleau, université LAVAL

[2] Science.ch (2017) Physique Quantique Relativiste, Atomistique, 2. Equation de Klein-Gordon généralisée

[3] Pierre Labastie (2010-11), MECANIQUE QUANTIQUE, L3 physique fondamentale, premier semestre 2010-11

[4] Michel Le Bellac (2003), Introduction à l'information quantique, Cours donné à l'Ecole Supérieur de Science Informatique (ESSI) Prétirage INLN 2003/08, page 10

[5] Eric Gourgoulhon (2013-2014), Relativité Générale, Observatoire de Paris, Master Astronomie Astrophysique et Ingénierie Spatiale année M2 parcours de recherche, Ondes gravitationnelles page 138 à 145

[6] Yurij V. Baryshev (2017), Fondation of relativistic astrophysics : Curvature of Riemannian Space versus Relativistic Quantum Field in Minkowski Space, Arxiv 1702.02020v1, page 17, $18,28-30$ 